\title{
Can histone epigenetic profiling become an alternative treatment modality for colon cancer?
}

\begin{abstract}
Colon cancer is one of the deadliest cancers which and second leading cause of cancerrelated deaths in the United State. It is third most common cancer in men and second most common cancer in women with an incidence of 570,000 per year. Advancement in early stages of diagnosis and treatment has enabled clinicians to extend and save the lives of many patients but the prognosis for patients with advanced disease or systemic metastasis is still too little. Adenomatous polyps, noncancerous (benign) tumors are the important cause for colon cancer which forms on the inner walls of the large intestine. They may grow into malignant colon cancers over time if they are not removed during colonoscopy. The cancer cells in the colon then march into and damage healthy tissue that is near the tumor, causing many complications subsequently moving on to metastasis invading and destroying other healthy tissues throughout the body which is very difficult to treat. This review is an attempt to find the possibility of development of alternative treatment modality for CRC using histone protein profiling.
\end{abstract}

Volume 7 Issue 2 - 2018

Thulasi G Pillai

Indian Institute of Technology Bombay, India

Correspondence: Thulasi G Pillai, Indian Institute of Technology Bombay, Powai, Mumbai-400076, Maharashtra, India, Email thulasigpillai@gmail.com

Received: January 13,2018 | Published: April 19, 2018

Keywords: colorectal cancer, histone, protein profiling, treatment

\section{Introduction}

Colorectal cancer (CRC) is a formidable health problem worldwide Almost $60 \%$ of cases are encountered in developed countries. In India, the annual incidence rates (AARs) for colon cancer in men are 4.4 per 100000 . The AAR for colon cancer in women is 3.9per 100000 (GLOBO. CAN 2008). There were marked developments in the chemotherapy of CRC in the last decade. Eight new drugs were approved for chemotherapy. The survival has doubled compared to 5-fluorouracil alone. KRAS testing opened an era of personalised therapy. Though on an average there are 90 genes mutated per cancer, only less than 20 pathways will actually drive cancer development. The available therapy with combinations for CRC is very expensive coming to $\$ 300000$ for 24 months. Early detection of tumors and improved therapy has lead to improvement in survival. With the administration of the adjuvant 5-fluorouracil, an improvement in survival of approximately $30 \%$, with an additional $20 \%$ improvement with the addition of oxaliplatin in stage III colon cancer is reported. Family history of CRC contributes only $25 \%$ thus most commonly occurs sporadically, suggesting a contribution for shared genes and environment. About 5-\% are from inherited mutations, while the remaining of the familial forms results from gene environment interactions.

Both, Human genome project and human proteome atlas has contributed remarkably for our increase in understanding of cancer Early diagnosis of CRC will help greatly in survival of patients. Limited research has been done in developing early stage diagnostic biomarker from human blood samples. The studies which were conducted were focussed on using miRNA as biomarker for early diagnosis of CRC. Some of them were effective for monitoring patients with metastatic disease (eg. CA 125 PSA) or monitoring relapse from chemotherapy (eg. CEA, PSA). The miRNA as biomarker succeeded some criteria needed for the tumor marker to be accepted for clinical practice such as stability in extracellular fluids and non invasive nature. It was very expensive with low throughput and could not be taken to routine clinical diagnosis. The conventional circulatory tumor markers were failed to a great extends due to their low specificity to detect malignancy. So the development of an early stage diagnostic maker which satisfies the requirements of an ideal biomarker (specific, non invasive, sensitive, stable in the sample and inexpensive) is a compelling emergency. APC promoter methylation is a frequent epigenetic alteration in colorectal cancer metastasis. ${ }^{1}$ Histone modifications and its influence on disease progression is also study of interest.

\section{Progression of CRC}

Normal colorectal epithelium $\longmapsto$ early adenoma $\longrightarrow$ Intermediate adenoma $\longrightarrow$ Advanced adenoma $\Longrightarrow$ colorectal carcinoma $\square$ Invasive carcinoma $\longrightarrow$ Metastatic carcinoma Development of an alternative treatment modality for CRC which is non invasive, riskless and without side effects is a necessity. The current treatment modalities of CRC are surgery, chemotherapy, and radiotherapy and chemo radiation. All these modalities are not free from side-effects. Most antineoplastic drugs induce various types of DNA damage: bridges, adducts, strand breaks or base lesions. ${ }^{2}$ The administration of chemotherapy in combination with radiotherapy can have an additive effect, in which there is no interaction between the treatment modalities and each strategy is separately effective. More promising is combinations in which a synergistic effect is achieved; the cytotoxic effect of the combination is greater than the sum of the effects of radiotherapy and chemotherapy alone as sensitization is involved in it mostly platinum adduct which interfere with repair through a mechanism known as steric hindrance.

Radiotherapy, either pre- or postoperatively induces late effects in the gastrointestinal tract. In the pelvis, the small bowel is the most radiosensitive and dose-limiting organ. Trials showed significantly more small bowel complications in the postoperatively irradiated patients $(11 \%)$ than in the preoperatively treated patients $(5 \%)$. In the non-irradiated patients, $6 \%$ small bowel obstructions were observed, 
demonstrating that postoperative radiotherapy leads to smaller bowel toxicity, whereas short-term preoperative radiotherapy has a similar toxicity as surgery alone. ${ }^{3}$

In $\mathrm{CRC}$, histone marks of aberrant acetylation and methylation levels on specific residues occur along with a plethora of deregulated enzymes that catalyze these reactions. Mutations, deletions or altered expression patterns alter the function of several histonemodifying proteins, supporting the vital role of epigenetic effectors in CRC oncogenesis, being closely associated to inactivation of tumor suppressor genes. ${ }^{4}$ In CRC, histone marks of aberrant acetylation and methylation levels on specific residues have been revealed, along with a plethora of deregulated enzymes that catalyze these reactions. The underlying mechanisms of the emerging epigenetic interplay along with the chemical compounds that are candidates for clinical use offer new insights for further investigation of key histone enzymes and new therapeutic targets.

Unlike genetic mutation, histone modifications are vibrant and reversible in nature and occur in a targeted manner by sitespecific modifiers. ${ }^{5}$ This specificity generates the potential for targeted inhibition of the specific histone modifiers, e.g. HDAC and HAT which in turn modulate tumor growth, inducing apoptosis and indirectly regulating other genomic output through variety of mechanisms. HDAC inhibitors (HDACi) have the potential to be used in the treatment of cancer. HDACi act synergistically or in an additive manner with different anticancer agents such as radiation therapy, chemotherapy, and differentiation agents. Chemotherapeutic agents which have additive or synergistic effects with HDACi therapy include antitubulin agent docetaxel; topoisomerase II inhibitors doxorubicin, etoposide, and ellipticine; and DNA cross-linking reagent, cisplatin. ${ }^{5}$ Histone targeting drugs, such as, histone deacetylase (HDAC) inhibitors, suberoylanilide hydroxamic acid (SAHA, Zolinza), and romidepsin (Istodax) have already been approved for clinical use by the FDA, and many more continue to be developed and evaluated. The first HDACi to be approved for clinical use in treating patients with cutaneous T-cell lymphoma is Vorinostat. Likewise, importance of HAT has been reported in B cell non-Hodgkin lymphoma that majorly occurred due to inactivating mutations in acetyl transferase genes causing reduction in HAT dosage. Interestingly, some of HAT inhibitors (HATi) have been shown to prevent growth of cancer cells.

Despite different available methods for colorectal cancer (CRC) screening and their proven benefits, morbidity, and mortality of this malignancy are still high, partly due to low compliance with screening. Minimally invasive tests based on the analysis of blood specimens can overcome this problem. Excellent trials are critical to do things better than we had in the past and what we have now to improve the survival of the patients with CRC. Using advanced technologies in genomics and proteomics, we believe that we will be able to get translated from bench to bedside translating the promise into reality in colon rectal carcinoma.

Genomic and epigenetic alterations are common in CRC. Chromosomal instability is regarded as a prerequisite for the stepwise development of the 'suppressor' pathway in which mutation of APC is the initiating event. The two recognised pathways to carcinogenesis in CRC are chromosomal instability pathway (CIP) and microsatellite instability pathway (MIP).

\section{Causes}

\section{a. Polyps}

Colon cancer usually derives from precancerous polyps that exist in the large intestine. It can be

i. Adenomas: can become cancerous but are usually removed during colonoscopy.

ii. Hyperplastic polyps: rarely become colon cancer.

iii. Inflammatory polyps: usually occur after inflammation of the colon (colitis) and may become cancerous.

\section{b. Mutation}

\section{c. Hereditary factors}

Cancer can be the result of a genetic predisposition that is inherited from family members. It is possible to be born with certain genetic mutations or a fault in a gene that makes one statistically more likely to develop cancer later in life.

\section{d. Other factors}

About $90 \%$ of the diagnosed cases are aged over 50. People with sedentary lifestyles, food with low fibre content.

\section{e. Other medical factors}

There are several diseases and conditions that have been associated with an increased risk of colon cancer. Diabetes, acromegaly (a growth hormone disorder), radiation treatment for other cancers, ulcerative colitis, and Crohn's disease all increase the risk of colon cancer.

\section{Epigenetic factors}

Epigenetic factors are important in underlying cancer development and progression. Epigenetic messages in chromatin include covalent modifications (acetylation, methylation, phosphorylation, and ubiquitination) of core nucleosomal proteins (histones). Histone modifications and other epigenetic mechanisms appear to work together in establishing and maintaining gene activity states, thus regulating a wide range of cellular processes. Molecules that modifies histone and protein complexes are discovered in last decade. Alterations in the function of histone-modifying complexes are believed to disrupt the pattern and levels of histone marks and consequently deregulate the control of chromatin-based processes, ultimately leading to oncogenic transformation and the development of cancer. ${ }^{6}$ The epigenetic information in the chromatin, also called as the histone code, has been proposed to extend and modulate the genetic (DNA) code in the regulation of key cellular processes. ${ }^{7}$ This code is believed to be 'read' by cellular machineries to regulate accessibility to, and functions of, chromatin DNA and the disruption of this code may lead to diseases, notably cancer.

Histone modifications function either by disrupting chromatin contacts or by affecting the recruitment of nonhistone proteins to chromatin. Their existence on histones can dictate the higher-order chromatin structure in which DNA is packaged and can orchestrate the ordered recruitment of enzyme complexes to manipulate DNA. So, histone modifications have the potential to influence many fundamental biological processes, some of which may be epigenetically inherited. ${ }^{8}$ Research on histone variants is a new hotspot and a new annotation 
of "histone code" in epigenetics. Histone exchange also reveals new changing mechanism of DNA-histone interaction. ${ }^{9}$

The genetic phenotype, 50 to $70 \%$ of all sporadic colorectal carcinomas, is characterised by a chromosomal instability (CIN) with the classical adenoma-carcinoma sequence due to alteration of the APC- $\beta$ catenin pathway with $\mathrm{p} 53$ mutations, SMAD alterations and $\mathrm{LOH}$ (loss of heterozygositiy) of 5q, $17 \mathrm{p} \mathrm{18q.} \mathrm{The} \mathrm{CpG} \mathrm{island}$ methylator phenotype (CIMP+) was described with an epigenetic inactivation of tumour suppressor genes that are typically inactivated by germline mutations in familiar cancer syndromes, e. g., Rb, VHL, hMLH1, p16 or BRCA. In colorectal cancer metastasis, APC promoter methylation is a frequent epigenetic alteration. ${ }^{1}$

In $\mathrm{CRC}$, histone marks of aberrant acetylation and methylation levels on specific residues occur along with an overabundance of deregulated enzymes that catalyze these reactions. Mutations, deletions or altered expression patterns change the function of several histone-modifying proteins, supporting the crucial role of epigenetic effectors in CRC oncogenesis, being closely associated to inactivation of tumor suppressor genes. ${ }^{4}$

Unlike genetic mutation, histone modifications are dynamic and reversible in nature and occur in a targeted manner by site-specific modifiers. ${ }^{5}$ The specificity generates the potential for targeted inhibition of the specific histone modifiers, e.g. HDAC and HAT which in turn modulate tumor growth, inducing apoptosis and indirectly regulating other genomic output through variety of mechanisms. HDAC inhibitors (HDACi) have the potential to be utilised in the treatment of cancer. HDACi act synergistically or in an additive manner with different anticancer agents such as radiation therapy, chemotherapy, and differentiation agents. Chemotherapeutic agents like antitubulin agent docetaxel; topoisomerase II inhibitors doxorubicin, etoposide, and ellipticine; and DNA cross-linking reagent, cisplatin ${ }^{5}$ have additive or synergistic effects with HDACi therapy. Histone targeting drugs, like histone deacetylase (HDAC) inhibitors, suberoylanilide hydroxamic acid (SAHA, Zolinza), and romidepsin (Istodax) have already been approved for clinical use by the FDA, and many more continue to be developed and evaluated. The first HDACi to be approved for clinical use in treating patients with cutaneous T-cell lymphoma is Vorinostat. The importance of HAT has been reported in B cell non-Hodgkin lymphoma that majorly occurred due to inactivating mutations in acetyl transferase genes causing reduction in HAT dosage. Some of HAT inhibitors (HATi) have been shown to prevent growth of cancer cells. C1A preferentially inhibits HDAC6 and modulates HDAC6 downstream targets leading to growth inhibition of a diverse set of cancer cell lines. ${ }^{10}$ HDAC6 has been recently found as an attractive target for the treatment of cancer. ${ }^{11-14}$ Certain limitations are also faced in the development of HDAC6 as a selective inhibitor. The HDAC6 selective inhibitors (tubacin, tubastatin A) have only contributed to validate HDAC6 as a target, preventing them from further pre-clinical and clinical development due to their unfavourable pharmacokinetic (PK) profiles, making them only good research tools. ${ }^{15,16}$

Local recurrences are one of the main problems in the treatment of rectal cancer. Local recurrence rates vary widely with reported incidences from 5 to $45 \%{ }^{17,18}$ Surgical resection remains the main treatment. Adjuvant modalities have their place in selected patients: cryotherapy, radiofrequency therapy, adjuvant chemotherapy portal vein embolisation or isolated liver perfusion. In chemotherapy most antineoplastic drugs induce various types of DNA damage: bridges, adducts, strand breaks or base lesions. ${ }^{2}$ Radiotherapy induces late effects in the gastrointestinal tract. The factors predisposing to late radiation-induced small bowel complications are total dose, dose per fraction, volume of irradiated small bowel and previous abdominal surgery. ${ }^{19}$ In the non-irradiated patients, $6 \%$ small bowel obstructions were observed, demonstrating that postoperative radiotherapy leads to more small bowel toxicity, whereas short-term preoperative radiotherapy has a similar toxicity as surgery alone. ${ }^{3}$

\section{Conclusion}

The available treatment modalities for colon cancer have severe side effects which makes the patient more weak. Development of alternative treatment modalities without side effects is a compelling emergency. Future expression profiling studies will give vast amounts of information on specific aspects of individual cases and should lead to tailored therapies or prevention programmes. These advances can facilitate the development of novel strategies to prevent, diagnose, and treat human colon malignancy. These developments will have farreaching public health consequences with knowledge at the brink of this fascinating era.

\section{Acknowledgment}

None.

\section{Conflict of interest}

Author declares there is conflict of interest.

\section{References}

1. Chen J, Röcken C, Lofton-Day C, et al. Molecular analysis of APC promoter methylation and protein expression in colorectal cancer metastasis. Carcinogenesis. 2005;26(1):37-43.

2. Nygren P. What is cancer chemotherapy? Acta Oncol 2001;40(2-3):166174.

3. Frykholm G, Glimelius B, Pahlman L. Preoperative or postoperative irradiation in adenocarcinoma of the rectum: final treatment results of a randomized trial and an evaluation of late secondary e $\square$ ects. Dis Colon Rectum. 1993;36(6):564-572.

4. Gargalionis AN1, Piperi C, Adamopoulos C, et al. Histone modifications as a pathogenic mechanism of colorectal tumorigenesis. Int J Biochem Cell Biol. 2012;44(8):1276-1289.

5. Tyagi M, Shafqat A Khan, Gupta S. Histone Post-translational Modifications as biomarks in cancer: Dream or Reality? Proteomics Society India. 2014;2:7-8.

6. Sawan C, Herceg Z. Histone modifications and cancer. Adv Genet. 2010;70:57-85.

7. Herceg Z. Epigenetic information in chromatin and cancer. Eur $J$ Cancer. 2009;45(Suppl 1):442-444.

8. Kouzarides T. Chromatin modifications and their function. Cell. 2007;128(4):693-705.

9. Wu N, Gui JF. Histone variants and histone exchange. Yi Chuan. 2006; 28(4):493-500.

10. Kaliszczak M, Trousil S, Åberg O, et al. A novel small molecule hydroxamate preferentially inhibits HDAC6 activity and tumour growth. Br J Cancer. 2013;108(2):342-350.

11. Boyault C, Sadoul K, Pabion M, et al. HDAC6, at the crossroads between cytoskeleton and cell signaling by acetylation and ubiquitination. Oncogene. 2007;26(37): 5468-5476. 
12. Lee YS, Lim KH, Guo X, et al. The cytoplasmic deacetylase HDAC6 is required for efficient oncogenic tumorigenesis. Cancer Res. 2008;68(18):7561-7569.

13. Kawada J, Zou P, Mazitschek R, et al. Tubacin kills Epstein-Barr virus (EBV)-Burkitt lymphoma cells by inducing reactive oxygen species and EBV lymphoblastoid cells by inducing apoptosis. J Biol Chem. 2009;284(25):17102-17109.

14. Rivieccio MA, Brochier C, Willis DE, et al. HDAC6 is a target for protection and regeneration following injury in the nervous system. Proc Nat Acad Sci USA. 2009;106(46):19599-19604.

15. Haggarty SJ, Koeller KM, Wong JC, et al. Domain-selective smallmolecule inhibitor of histone deacetylase 6 (HDAC6)-mediated tubulin deacetylation. Proc Natl Acad Sci USA. 2003;100(8):4389-4394.
16. Butler $\mathrm{KV}$, Kalin J, Brochier $\mathrm{C}$, et al. Rational design and simple chemistry yield a superior, neuroprotective HDAC6 inhibitor, tubastatin A. J Am Chem Soc. 2010;132(31):10842-10846.

17. Harnsberger JR, Vernava VM, Longo WE. Radical abdominopelvic lymphadenectomy: historic perspective and current role in the surgical management of rectal cancer. Dis Colon Rectum. 1994;37(1):73-87.

18. MacFarlane JK, Ryall RD, Heald RJ. Mesorectal excision for rectal cancer. Lancet. 1993;341(8843):457-460.

19. Letschert J. The prevention of radiation-induced small bowel complications. Eur J Cancer. 1995;31A(7-8):1361-1365. 